\title{
Interference-Aware Resource Control in Multi-Antenna Cognitive Ad Hoc Networks with Heterogeneous Delay Constraints
}

\author{
Xiaoming Chen, Member, IEEE, and Hsiao-Hwa Chen, Fellow, IEEE
}

\begin{abstract}
In this work, we consider a multi-antenna cognitive ad hoc network (CAHNet) with heterogeneous delay requirements. To fulfill the interference and delay constraints simultaneously, we propose to perform adaptive zero-forcing beamforming (ZFBF) at cognitive transmitters according to interference channel state information (CSI). To assist the CAHNet to obtain the interference CSI, we use a win-win inter-network cooperation strategy, namely quantized interference CSI feedback from the primary network to CAHNet through a feedback link, under the condition that the CAHNet pays a proper price for it. Considering the scarcity of feedback and power resources, we focus on the minimization of the overall resource cost subject to both interference and delay constraints. To solve the problem, we derive a joint feedback and power control algorithm amongst multiple links of CAHNet. Finally, simulation results validate the effectiveness of the proposed algorithm.
\end{abstract}

Index Terms-Cognitive network; ad hoc network; multiantenna; resource control

\section{INTRODUCTION}

Wireless communication with a given delay guarantee is a challenging issue, especially in cognitive networks. On one hand, more resources, such as power and spectrum, should be allocated to satisfy the delay requirement over a fading channel. On the other hand, the utilization of the resource is strictly limited due to spectrum scarcity and interference constraint in a cognitive network. Therefore, resource allocation in a cognitive network with a given delay guarantee receives a lot of attention.

Recently, cognitive ad hoc network (CAHNet) has become an active research topic due to its ability of self-adaptation to wireless channel conditions, self-exploration of available spectrum, and self-organization of ubiquitous interconnection [1] [2]. Resource control in a CAHNet with a delay constraint poses a great challenge different from those in a normal cognitive network. Let us take a look at a simple example. Since aggregated interference to a primary network is determined by multiple links of CAHNet, power control works based not only on the interference constraint, but also

This work was supported by the grants from the NUAA Research Funding (NN2012004), the open research fund of State Key Laboratory of Integrated Services Networks, Xidian University (No. ISN14-01), and Taiwan National Science Council (NSC99-2221-E-006-016-MY3).

Xiaoming Chen (e-mail: chenxiaomingenuaa.edu.cn) is with the College of Electronic and Information Engineering, Nanjing University of Aeronautics and Astronautics, and also with the State Key Laboratory of Integrated Services Networks, Xidian University, China. Hsiao-Hwa Chen (e-mail: hshwchen@ieee.org) is with the Department of Engineering Science, National Cheng Kung University, Tainan, 70101 Taiwan. on the interaction of different links. Specifically, if one link is allowed to produce more interference, it can use high power to meet its delay requirement, while the other links must lower the power due to a small interference proportion for a given total interference constraint. In other words, the power control should take into account heterogeneous delay requirements. However, it seems to be difficult to satisfy the delay requirements by power control alone in a CAHNet, because transmit power is confined by an interference upper bound. Inspired by interference cancellation in multi-antenna systems, it is found that the performance of a multi-antenna cognitive network can be further improved by exploiting the spatial degrees of freedom. If a cognitive network has perfect interference CSI, the interference can be cancelled completely or confined within a required range by using a proper transmit beam [3]. In [4], the authors presented an optimal beam design method to maximize the spectrum efficiency, while satisfying the interference constraint. If a cognitive transmitter has only partial interference CSI, the authors in [5] and [6] proved that it is also beneficial to improve the performance through robust beamforming. However, the previous works did not solve the problems on how a cognitive network obtains the interference CSI and what is the relationship between the performance and the amount of interference CSI in a cognitive network.

In this letter, we propose to perform adaptive beamforming at multiple cognitive transmitters based on the interference CSI to satisfy both interference and delay requirements. Especially, we suggest to use limited cooperation between two networks to achieve a win-win situation accordingly. Specifically, a CAHNet purchases some quantized interference CSI from a primary network at a proper price, so that the CAHNet fulfills the delay requirement and the primary network gets some rewards. Furthermore, we reveal the relationship between the inter-network interference, delay requirement, feedback bits, and transmit power. Then, via minimizing the overall resource cost while satisfying interference and delay requirements, we derive a joint feedback and power control algorithm, which can achieve the same effect as an optimal exhaustive search algorithm, but at a relatively low complexity.

The rest of this letter is outlined as follows. Section II gives a brief introduction of a multi-antenna CAHNet with heterogeneous delay requirements. Section III focuses on the design of a joint feedback and power control algorithm, and Section IV presents the numerical results to validate the performance of the proposed algorithm. Finally, Section V summarizes the findings obtained in this letter. 


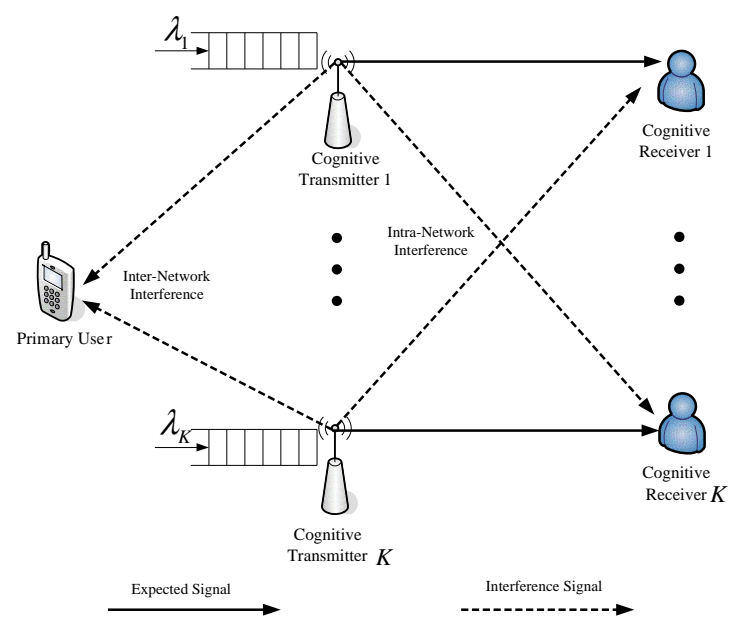

Fig. 1. An overview of a cognitive ad hoc network model.

\section{SySTEM MODEL}

Let us consider a cognitive ad hoc network (CAHNet) consisting of $K$ transmitter-receiver links coexisting with a primary network, as depicted in Fig. 11 For ease of analysis, we assume that each cognitive transmitter equips with $N_{t}$ antennas and each cognitive receiver has one antenna. We also assume that the primary base station is far from the CAHNet, so we can omit the primary base station here. A primary user deploys one antenna due to the size limitation. We use $\mathbf{h}_{j, i}$ to denote the channel from cognitive transmitter $i$ to cognitive receiver $j$, and $\mathbf{g}_{0, i}$ to denote the channel from cognitive transmitter $i$ to the primary user. Assume that all the channels remain constant during a transmission interval and suffer independent fading on an interval by interval basis according to a circular symmetry complex gaussian distribution with zero mean and variance $\sigma_{j, i}^{2}$, where $i=1, \cdots, K$ and $j=0, \cdots, K$. At the beginning of each transmission interval, cognitive transmitters obtain partial interference CSI from the primary user by using a codebook-based limited feedback scheme [6]. Specifically, the primary user obtains the instantaneous interference CSI via channel estimation, and selects an optimal quantized codeword for the interference channel $\mathbf{g}_{0, i}$ from a predetermined quantization codebook $\mathcal{G}_{i}=\left\{\hat{\mathbf{g}}_{i, 1}, \cdots, \hat{\mathbf{g}}_{i, 2^{B_{i}}}\right\}$ of size $2^{B_{i}}$ with a criterion such that $l=\arg \max _{\hat{\mathbf{g}}_{i, l} \in \mathcal{G}_{i}}\left|\tilde{\mathbf{g}}_{0, i}^{H} \hat{\mathbf{g}}_{i, l}\right|^{2}$, where $\tilde{\mathbf{g}}_{0, i}=\frac{\mathbf{g}_{0, i}}{\left\|\mathbf{g}_{0, i}\right\|}$ is the corresponding channel direction. Then, the primary user conveys index $l$ to cognitive transmitter $i$ with $B_{i}$ bits via a feedback link, and cognitive transmitter $i$ recovers the quantized CSI from the same codebook. In a CAHNet, we assume that cognitive transmitter $i$ may interfere at most $L$ closest cognitive receivers, and it can obtain full directional information of these intra-network interference channels $\tilde{\mathbf{h}}_{i_{j}, i}$, where $j=1, \cdots, L$, through an intra-network information exchange mechanism. For example, the cognitive receiver estimates the intra-network interference CSI and conveys it to a central control node, which delivers the CSI to the corresponding cognitive transmitter. Based on the information of inter-network and intra-network interference channels, cognitive transmitter $i$ determines its optimal transmit beam $\mathbf{w}_{i}$ making use of zero-force beamforming (ZFBF) method, such that we have

$$
\begin{aligned}
\hat{\mathbf{g}}_{i, \mathrm{opt}}^{H} \mathbf{w}_{i} & =0, \\
\mathbf{h}_{i_{j}, i}^{H} \mathbf{w}_{i} & =0,
\end{aligned}
$$

where $\hat{\mathbf{g}}_{i, \text { opt }}$ is the optimal quantized inter-network interference CSI related to cognitive transmitter $i$ for a given transmission interval.

In such a CAHNet, all cognitive links require different transmission services. The data for link $i$ arrives in the form of packet of a fixed length $N_{b}$ bits with its average arrival rate $\lambda_{i}$ (packets/interval), and it has the minimum average delay requirement $D_{i}$ (intervals) related to its service style. Following [7], in order to satisfy the delay constraint we know that the average transmission rate should meet the following condition:

$$
\bar{R}_{i} \geq \frac{\left(2 D_{i} \lambda_{i}+2\right)+\sqrt{\left(2 D_{i} \lambda_{i}+2\right)^{2}-8 D_{i} \lambda_{i}}}{4 D_{i}} \frac{N_{b}}{T}=\bar{r}_{i},
$$

where $T$ is the length of a transmission interval.

The precondition that a CAHNet is allowed to access the licensed spectrum is that the aggregated inter-network interference must satisfy a given constraint. It was proved that an average interference constraint (AIC) is more beneficial to improve the performance of a cognitive network than a peak interference constraint (PIC) [8]. Thus, similar to [9], we take AIC as the interference constraint. In order to realize delay guaranteed transmission at a minimum cost, this work focuses on the minimization of the overall resource cost subject to both interference and delay constraints by jointly optimizing feedback bits $B_{i}$ and transmit power $P_{i}$ for $i=1, \cdots, K$ according to the interference CSI, delay requirements, and arrival rates.

\section{Delay Driven Joint Resource Control}

As interference constraint is the precondition, prior to discussing joint resource control, we first perform an investigation on the relationship of the average aggregated interference, transmit power, and feedback bits.

According to the theory of random vector quantization [10], the relationship between the original and quantized interference channel direction vectors can be expressed as

$$
\tilde{\mathbf{g}}_{0, i}=\sqrt{1-a_{0, i}} \hat{\mathbf{g}}_{i, \mathrm{opt}}+\sqrt{a_{0, i}} \mathbf{s}_{0, i}
$$

where $\mathbf{s}_{0, i}$ is the quantization error due to finite quantization bits, which is an unit norm vector isotropically distributed in the null-space of $\hat{\mathbf{g}}_{i, \mathrm{opt}}$, and is independent of $a_{0, i} \cdot a_{0, i}=$ $\sin ^{2}\left(\angle\left(\tilde{\mathbf{g}}_{0, i}, \hat{\mathbf{g}}_{i, \text { opt }}\right)\right)$ is the magnitude of the quantization error. Therefore, the inter-network interference caused by cognitive transmitter $i$ is given by

$$
\left|\sqrt{P_{i}} \mathbf{g}_{0, i}^{H} \mathbf{w}_{i}\right|^{2}=P_{i}\left\|\mathbf{g}_{0, i}\right\|^{2} a_{0, i}\left|\mathbf{s}_{0, i}^{H} \mathbf{w}_{i}\right|^{2},
$$

which holds because of (1). The sum average interference can be expressed as

$$
\bar{I}=\sum_{i=1}^{K} P_{i} E\left[\left\|\mathbf{g}_{0, i}\right\|^{2}\right] E\left[a_{0, i}\right] E\left[\left|\mathbf{s}_{0, i}^{H} \mathbf{w}_{i}\right|^{2}\right],
$$


which follows due to the fact that the magnitude and direction of a channel are independent of each other. Examining the right hand side of (6), we can find that $\left\|\mathbf{g}_{0, i}\right\|^{2}$ is $\sigma_{0, i}^{2} \chi^{2}\left(2 N_{t}\right)$ distributed, so that we have $E\left[\left\|\mathbf{g}_{0, i}\right\|^{2}\right]=N_{t} \sigma_{0, i}^{2}$. According to the definition of quantization error, $a_{0, i}$ is equal to $1-\left|\tilde{\mathbf{g}}_{0, i}^{H} \hat{\mathbf{g}}_{i, \mathrm{opt}}\right|^{2}$. For an arbitrary quantization codeword $\hat{\mathbf{g}}_{i, l}$, $1-\left|\tilde{\mathbf{g}}_{0, i}^{H} \hat{\mathbf{g}}_{i, l}\right|^{2}$ is $\beta\left(N_{t}-1,1\right)$ distributed, so that $a_{0, i}$ is the minimum of $2^{B_{i}}$ independent $\beta\left(N_{t}-1,1\right)$ random variables, and thus its expectation can be tightly upper bounded as $E\left[a_{0, i}\right]<2^{-\frac{B_{i}}{N_{t}-1}}$. Finally, since $\mathbf{s}_{0, i}$ and $\mathbf{w}_{i}$ are i.i.d. isotropic vectors in a $\left(N_{t}-1\right)$ dimensional null-space of $\hat{\mathbf{g}}_{i, \mathrm{opt}},\left|\mathbf{s}_{0, i}^{H} \mathbf{w}_{i}\right|^{2}$ is $\beta\left(1, N_{t}-2\right)$ distributed, whose expectation is equal to $\frac{1}{N_{t}-1}$. As a result, we have $\bar{I}<\sum_{i=1}^{K} \frac{\sigma_{0, i}^{2} P_{i} N_{t}}{N_{t}-1} 2^{-\frac{B_{i}}{N_{t}-1}}$. In other words, for a given AIC $\bar{I}_{\text {AIC }}$, the power and feedback joint control must satisfy $\sum_{i=1}^{K} \frac{\sigma_{0, i}^{2} P_{i} N_{t}}{N_{t}-1} 2^{-\frac{B_{i}}{N_{t}-1}} \leq \bar{I}_{\text {AIC }}$.

For cognitive link $i$, the receive signal can be expressed as

$$
\begin{aligned}
y_{i} & =\sqrt{P_{i}} \mathbf{h}_{i, i}^{H} \mathbf{w}_{i} x_{i}+\sum_{j=1}^{L} \mathbf{h}_{i, i_{j}}^{H} \mathbf{w}_{i_{j}} x_{i_{j}}+n_{i} \\
& =\sqrt{P_{i}} \mathbf{h}_{i, i}^{H} \mathbf{w}_{i} x_{i}+n_{i},
\end{aligned}
$$

where $x_{i}$ is the normalized transmit signal of cognitive transmitter $i, n_{i}$ is additive white Gaussian noise with zero mean and unit variance. (7) follows from (2). Hence, the receive SNR and the corresponding transmission rate are $\gamma_{i}=P_{i}\left|\mathbf{h}_{i, i}^{H} \mathbf{w}_{i}\right|^{2}$ and $R_{i}=\log _{2}\left(1+\gamma_{i}\right)$, respectively. Since $\mathbf{w}_{i}$ is a normalized vector independent of $\mathbf{h}_{i, i}, \gamma_{i}$ is $P_{i} \sigma_{i, i}^{2} \chi^{2}(2)$ distributed. In this context, the average transmission rate for cognitive link $i$ can be computed as

$$
\begin{aligned}
\bar{R}_{i} & =W \int_{0}^{\infty} \log _{2}(1+x) \frac{\exp \left(-\frac{x}{P_{i} \sigma_{i, i}^{2}}\right)}{P_{i} \sigma_{i, i}^{2}} d x \\
& =W \log _{2}(e) \exp \left(\frac{1}{P_{i} \sigma_{i, i}^{2}}\right) E_{1}\left(\frac{1}{P_{i} \sigma_{i, i}^{2}}\right)
\end{aligned}
$$

where $W$ is the bandwidth of licensed spectrum, and $E_{1}(x)$ is the exponential-integral function of the first order. For a given arrival rate $\lambda_{i}$, in order to fulfill delay constraint $D_{i}$, average transmission rate $\bar{R}_{i}$ should not be less than $\bar{r}_{i}$, as shown in (3). Since $\bar{R}_{i}$ is an increasing function of $P_{i}$, the delay constraint is equivalent to $P_{i} \geq P_{i}^{\star}$, where $P_{i}^{\star}$ is the solution of the function $W \log _{2}(e) \exp \left(\frac{1}{x \sigma_{i, i}^{2}}\right) E_{1}\left(\frac{1}{x \sigma_{i, i}^{2}}\right)=\bar{r}_{i}$.

In a CAHNet, transmit power and feedback bits are two important and scarce resources, where transmit power affects terminal battery life time directly and feedback bits determine the cost. Thus, this letter focuses on the design of a joint power and feedback control algorithm by minimizing the overall resource cost while satisfying interference and delay constraint. Let $\mu$ be the feedback price per bit from a primary user to cognitive transmitters, which depends on the feedback cost consisting of the power and spectrum used for channel estimation, codeword selection and index conveyance, and $\varphi$ be the power utilization cost per watt at cognitive transmitters. Then, the joint resource control is equivalent to the following optimization problem:

$$
\begin{array}{ll}
J_{1}: & \min _{\mathbf{B}, \mathbf{P}} \sum_{i=1}^{K} \mu B_{i}+\varphi P_{i}, \\
\text { s.t. } & \sum_{i=1}^{K} \frac{\sigma_{0, i}^{2} P_{i} N_{t}}{N_{t}-1} 2^{-\frac{B_{i}}{N_{t}-1}} \leq \bar{I}_{\mathrm{AIC}}, \\
& P_{i} \geq P_{i}^{\star}, \quad i=1, \cdots, K,
\end{array}
$$

where $\mathbf{B}=\left\{B_{1}, \cdots, B_{K}\right\}^{T}$ and $\mathbf{P}=\left\{P_{1}, \cdots, P_{K}\right\}^{T}$ are $K$ dimensional feedback bits and transmit power vectors, respectively. (9) is the overall resource cost function, and (10) and (11) represent interference and delay constraints, respectively. Clearly, $J_{1}$ is a mixed integer programming problem, and it is difficult to get a close-form expression of optimal $\mathbf{B}$ and $\mathbf{P}$. Examining the constraint condition (10), we can find that the lower the power, the smaller the interference. Thus, we can use less bits to meet AIC. In other words, the resource cost is minimized when using the minimum power. As shown in (11), $P_{i}^{\star}$, where $i=1, \cdots, K$, are the lower bound on the power due to the delay constraint. Therefore, they give the optimal transmit power indeed. Under this condition, $J_{1}$ is reduced to

$$
\begin{array}{ll}
J_{2}: & \min _{\mathbf{B}} \sum_{i=1}^{K} B_{i}, \\
\text { s.t. } & \sum_{i=1}^{K} \frac{\sigma_{0, i}^{2} P_{i}^{\star} N_{t}}{N_{t}-1} 2^{-\frac{B_{i}}{N_{t}-1}} \leq \bar{I}_{\mathrm{AIC}},
\end{array}
$$

where $J_{2}$ is a pure feedback control problem. Intuitively, allocating more bits to a cognitive transmitter with a strong interference mitigation capability is beneficial to decrease the feedback cost. Inspired by this idea, we propose a greedy feedback control algorithm. First, we define feedback gain as the amount of interference reduction by adding one more bit feedback. For example, the feedback gain of cognitive transmitter $i$ with $B_{i}$ feedback bits at present is $\eta_{i}\left(B_{i}\right)=$ $\frac{\sigma_{0, i}^{2} P_{i}^{\star} N_{t}}{N_{t}-1}\left(2^{-\frac{B_{i}}{N_{t}-1}}-2^{-\frac{B_{i}+1}{N_{t}-1}}\right)$. Note that feedback gain is variable, as inter-network interference is not a linear function of feedback bits. Then, we can compare the feedback gains of all transmitters and add one more bit to the transmitter with the largest feedback gain. Repeat the process until the aggregated interference satisfies the AIC. The joint feedback and power control algorithm can be summarized as follows:

1) Initialization: With given $N_{t}, K, \bar{I}_{\mathrm{AIC}}, W, T, D_{i}, N_{b}$ and $\sigma_{j, i}^{2}$, set $\mathbf{P}=\mathbf{0}$ and $\mathbf{B}=\mathbf{0}$.

2) Compute $\bar{r}_{i}$, and then $P_{i}^{\star}$. $P_{i}=P_{i}^{\star}$ for $i=1, \cdots, K$ are the optimal transmit powers.

3) Let $\eta_{i}\left(B_{i}\right)=\frac{\sigma_{0, i}^{2} P_{i}^{\star} N_{t}}{N_{t}-1}\left(2^{-\frac{B_{i}}{N_{t}-1}}-2^{-\frac{B_{i}+1}{N_{t}-1}}\right)$. Search $i^{\star}=$ $\arg \max _{1 \leq i \leq K} \eta_{i}\left(B_{i}\right)$. Let $B_{i^{\star}}=B_{i^{\star}}+1$.

4) If $\sum_{i=1}^{K} \frac{\sigma_{0, i}^{2} P_{i}^{\star} N_{t}}{N_{t}-1} 2^{-\frac{B_{i}}{N_{t}-1}}>\bar{I}_{\mathrm{AIC}}$, then go to 3$)$.

Assume that the total feedback amount is $B$ bits. The computation complexity of the above greedy resource control algorithm scales with $K B$, while that of the optimal algorithm based on exhaustive search scales with $K^{B}$, so that the proposed algorithm has a much lower complexity, especially 
TABLE I

OPTIMAL NUMBER OF FEEDBACK BITS WITH DIFFERENT AICS.

\begin{tabular}{|c|c|c|c|c|}
\hline & & $B_{1}$ & $B_{2}$ & $B_{3}$ \\
\hline \multirow[b]{2}{*}{$\bar{I}_{\mathrm{AIC}}=0.01$} & $\mathrm{GA}$ & 1 & 6 & 9 \\
\hline & EA & 0 & 6 & 10 \\
\hline \multirow[b]{2}{*}{$\bar{I}_{\text {AIC }}=0.02$} & GA & 0 & 3 & 5 \\
\hline & EA & 0 & 2 & 6 \\
\hline \multirow[b]{2}{*}{$\bar{I}_{\mathrm{AIC}}=0.03$} & $\overline{\mathrm{GA}}$ & 0 & 1 & 3 \\
\hline & EA & 0 & 0 & 4 \\
\hline \multirow[b]{2}{*}{$\bar{I}_{\mathrm{AIC}}=0.04$} & $\mathrm{GA}$ & 0 & 0 & 1 \\
\hline & EA & 0 & 0 & 1 \\
\hline
\end{tabular}

with a larger $K$. In some extreme cases shown as follows, it is likely to obtain the optimal control algorithms more easily.

1) Loose AIC and/or long separation distance from a primary user: If AIC is sufficiently loose, so that the AIC can always be satisfied even when the design of transmit beam at a cognitive transmitter is independent of inter-network interference CSI. In other words, without interference CSI, a CAHNet can work well. Under this condition, $P_{i}=P_{i}^{\star}$ and $B_{i}=0$ for all $i$ are the optimal control algorithm. Furthermore, in the case of $P_{i}=P_{i}^{\star}$ and $B_{i}=0$, the aggregated average interference is $\bar{I}_{0}=\sum_{i=1}^{K} \frac{\sigma_{0, i}^{2} P_{i}^{\star} N_{t}}{N_{t}-1}$, such that when $\bar{I}_{\text {AIC }} \geq \bar{I}_{0}$, the above algorithm is optimal. Moreover, if the distance between CAHNet and a primary user is long enough, due to path loss, the aggregated average interference always satisfies AIC even with $B_{i}=0$. Let $\sigma_{0,1}^{2}=\cdots=\sigma_{0, K}^{2}=\sigma_{0}^{2}=d^{-\alpha}$, where $d$ denotes the distance and $\alpha$ denotes the path loss exponent. When $\sigma_{0}^{2} \leq \bar{I}_{\mathrm{AIC}} /\left(\frac{N_{t}}{N_{t}-1} \sum_{i=1}^{K} P_{i}^{\star}\right)$, the AIC is always met. Hence, when the minimum distance between cognitive transmitters and a primary user is not shorter than $d=\left(\bar{I}_{\mathrm{AIC}} /\left(\frac{N_{t}}{N_{t}-1} \sum_{i=1}^{K} P_{i}^{\star}\right)\right)^{1 / \alpha}, P_{i}=P_{i}^{\star}$ and $B_{i}=0$ for all $i$ are the optimal control algorithm.

2) $\bar{I}_{\mathrm{AIC}}=0$ : If a primary user can not bear any interference from the CAHNet, namely $\bar{I}_{\mathrm{AIC}}=0$, then a cognitive transmitter must know full interference CSI, and transmits its signal in the null-space of interference channel. In this case, $B_{i}$ must approach to infinity. However, it is impractical in any real CAHNet.

\section{NumericAl RESUlTS}

To evaluate the performance of the proposed algorithm, we present several numerical results with various AICs. We set $N_{t}=4, K=3, N_{b}=80, W=50 \mathrm{KHz}, \lambda_{1}=0.3, \lambda_{2}=0.4$, $\lambda_{3}=0.5, D_{1}=2, D_{2}=4, D_{3}=20, \sigma_{1,1}^{2}=\sigma_{2,2}^{2}=$ $\sigma_{3,3}^{2}=0.01, \sigma_{0,1}^{2}=0.0001, \sigma_{0,2}^{2}=0.0005, \sigma_{0,3}^{2}=0.0010$, $\mu_{1}=\mu_{2}=\mu_{3}=1$, and $T=5 \mathrm{~ms}$. In what follows, we use GA and EA to denote the proposed greedy algorithm and exhaustive search algorithm, respectively.

Table \ shows the feedback control results based on GA and EA with different AICs. As AIC becomes relatively loose, the required feedback bits decrease sharply. For example, there are 15 bits reduction when $\bar{I}_{\text {AIC }}$ changes from 0.01 to 0.04 . For each AIC, cognitive transmitter 3 is always allocated the most bits, since its feedback gain is the largest in most cases, which is consistent with the principle of the proposed greedy control algorithm. It is found that, although the control results are different with a given AIC, the total feedback bits are kept the same. In other words, the optimal control result is not unique, and GA is also optimal in the sense of resource cost. Moreover, Fig. 2 presents the average sum interference of GA and EA schemes under different AICs. It is found that GA has nearly the same average sum interference as EA. However, it has a quite lower complexity compared to the exhaustive search algorithm, which is more appealing in a practical CAHNet.

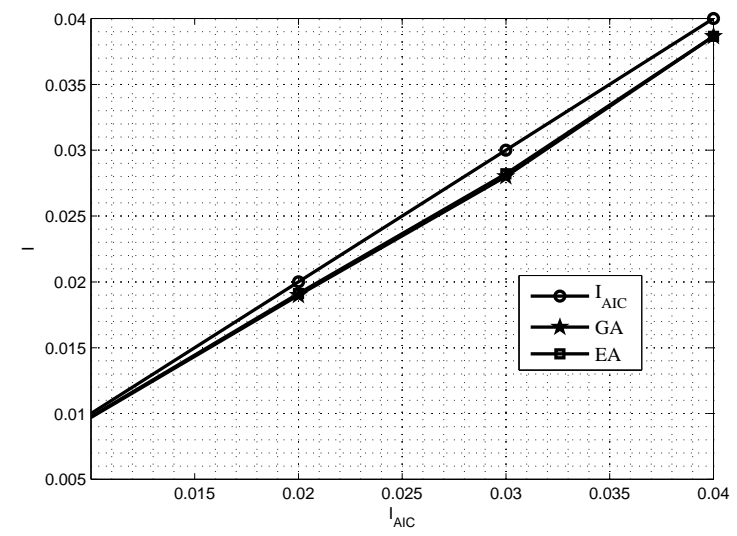

Fig. 2. Average interference of GA and EA schemes.

\section{CONCLUSION}

A major contribution of this letter is the introduction of the limited inter-network cooperation into a multi-antenna $\mathrm{CAH}$ Net to achieve a win-win situation. Considering the scarcity of feedback and power resources, we proposed a greedy resource control algorithm by minimizing the overall resource cost. The numerical results confirmed the effectiveness of the proposed algorithm.

\section{REFERENCES}

[1] I. F. Akyildiz, W-Y. Lee, and K. R. Chowdhury, "CRAHNs: cognitive radio ad hoc networks," Ad Hoc Networks, pp. 810-836, July 2009.

[2] X. Wang, A. Wong, and P. Ho, "Stochastic medium access for cognitive radio ad hoc networks," IEEE Journal on Sel. Areas in Commun., vol. 29, no. 4, pp. 770-783, Apr. 2011.

[3] M. Jung, K. Hwang, and S. Choi, "Interference minimization approach to precoding scheme in MIMO-based cognitive radio networks," IEEE Commun. Lett., vol. 15, no. 8, pp. 789-792, Aug. 2011.

[4] R. Zhang, and Y-C. Liang, "Exploiting multi-antennas for opportunistic spectrum sharing in cognitive radio networks," IEEE Journal of Sel. Topics in Signal Process., vol. 2, no. 1, pp. 88-102, Feb. 2008.

[5] H. Du, T. Ratnarajah, M. Pesavento, and C. B. Papadias, "Joint transceiver beamforming in MIMO cognitive radio network via second-order cone programming," IEEE Trans. Signal Process., vol. 60, no. 2, pp. 781-792, Feb. 2012.

[6] X. Chen, and C. Yuen, "Efficient resource allocation in rateless coded MU-MIMO cognitive radio network with QoS provisioning and limited feedback," IEEE Trans. Veh. Tech., vol. 62, no. 1, pp. 395-399, Jan. 2013.

[7] D. S. W. Hui, V. K. N. Lau, and W. H. Lam, "Cross-layer design for OFDMA wireless systems with heterogeneous delay requirements," IEEE Trans. Wireless Commun., vol. 6, no. 8, pp. 2872-2880, Aug. 2007.

[8] R. Zhang, "On peak versus average intererence power constraints for protecting primary users in cognitive radio networks," IEEE Trans. Wireless Commun., vol. 8, no. 4, pp. 2112-2120, Apr. 2009.

[9] C-X. Wang, X. Hong, H-H. Chen, and J. Thompson, "On capacity of cognitive radio networks with average interference power constraints," IEEE Trans. Wireless Commun., vol. 8, no. 4, pp. 1620-1625, Apr. 2009.

[10] N. Jindal, "MIMO Broadcast channels with finite-rate feedback," IEEE Trans. Infor. Theory, vol. 52, no. 11, pp. 5045-5060, Nov. 2006. 